\title{
ESTRATEGIAS NUTRICIONALES PARA LA COMPETICIÓN EN EL FÚTBOL
}

\author{
NUTRITIONAL STRATEGIES FOR \\ SOCCER PLAYING
}

\author{
José Antonio González J. (1), Inmaculada Cobos H. (2), Edgardo Molina S. (3)
}

(1) Facultad del Deporte. Universidad Pablo de Olavide. Sevilla. España.

(2) Enfermera de Dispositivo de Urgencias del Sistema Nacional de Salud, Universidad de Sevilla-España

(3) Departamento de Educación Física, Deporte y Recreación.

Universidad Metropolitana de Ciencias de la Educación. Santiago, Chile.

\begin{abstract}
Nutritional strategies are one of basic fundaments of performance in sports. In soccer, like in the majority of professional sports, nutritional control of players is very important. This work presents a general proposal of the structure of the diet for training and the competition based in caloric disassociation, to achieve muscular surcharge of carbohydrates. Nutritional strategies based in the disassociation of the diet, according to metabolic needs of the competition and the training have demonstrated a great efficacy in performance improvement of players. Numerous studies report about metabolic needs of soccer during a game and during training. It has been demonstrated than the super-compensation of carbohydrates is the most effective nutritional strategy.
\end{abstract}

Key words: nutrition, soccer, disassociation diet, carbohydrates, performance.

Este trabajo fue recibido el 7 de Julio de 2009 y aceptado para ser publicado el 12 de Enero de 2010.

\section{INTRODUCCION A LA NUTRICIÓN EN EL FÚTBOL}

El juego del fútbol requiere un elevado gasto energético producido, en parte, por la elevada distancia recorrida durante un partido. Conforme aumenta el nivel competitivo mayor es la intensidad a la que se realizan los esfuerzos y mayor es el número de partidos jugados por temporada. La realización por partido de unas 30 a 50 carreras, de 10 a 15 metros, de muy alta intensidad, es determinante para provocar una dramática reducción de las reservas energéticas del futbolista que deben ser reconstituidas gracias a una correcta alimentación.

Numerosos estudios realizados con tecnología GPS, demuestran que las distancias recorridas durante un partido de fútbol están en torno a los 10000 metros (1).

Para proporcionar estrategias nutricionales a jugadores de fútbol, es fundamental conocer las demandas energéticas y saber que sustratos se utilizan durante los partidos.

El glucógeno muscular es el sustrato más importante para los jugadores de fútbol. Numerosos estudios, ya clásicos, informaron de la importancia de los hidratos de carbono para el rendimiento de los futbolistas (2) y cómo los depósitos de glucógeno muscular eran casi totalmente agotados durante la segunda parte del partido. Posteriormente podemos encontrar muchas otras investigaciones que reportan resultados en este sentido $(3,4)$. En estudios muy recientes como el de Krustrup y colaboradores (5), se demostró que en la mayor parte de las fibras musculares del vasto lateral del cuádriceps, la depleción de los depósitos de glucógeno muscular al final del partido fue total o casi total.

Las demandas energéticas y metabólicas de los jugadores de fútbol durante los entrenamientos y los partidos varían a lo largo de la temporada, varían según los niveles de competición y según las características individuales de los jugadores. El coste de energía medio estimado para un entrenamiento o un partido en futbolistas de élite está alrededor de $1500 \mathrm{kcal}$ en hombres y $1000 \mathrm{kcal}$ en mujeres. Los futbolistas deberían comer una amplia variedad de alimentos que proporcione suficientes carbohidratos como combustible (6). 
En un estudio realizado por Leblanc y colaboradores $(7,8)$, informaron que el consumo calórico diario de futbolistas profesionales se encontraba en un rango que iba de $2352 \pm 454$ a $3395 \pm 396$, aporte calórico que se considera insuficiente si lo comparamos con las recomendaciones que estarían en un rango de 3819 a 5185 kcal al día.

Asimismo estos mismos autores reportaron que las dietas de estos futbolistas eran desequilibradas. Siendo muy excesivo el aporte calórico en base grasas, entre 29 y $34 \%$ frente al $20 \%$ recomendado, en detrimento de los hidratos de carbono. Resultados similares se encontraron en el reciente estudio realizado por Ruiz y colaboradores (8).

El objetivo de este trabajo fue presentar como tema de actualización una propuesta general de la estructura de la dieta para preparar la competición de futbolistas seniors, basada en la disociación calórica de la dieta, buscando una sobrecarga de hidratos de carbono.

La revisión documental se ha realizado mediante búsquedas en las siguientes bases de datos, (a las cuales se ha accedido desde los servicios de documentación de la Universidad Pablo de Olavide de Sevilla, España): MEDLINE (OvidSP), SCOPUS (Elsevier) y SPORTDISCUS (EbscoHost).

Los descriptores de búsqueda utilizados han sido: soccer, football, nutrition, glycogen, intake and carbohydrate.

\section{ESTRUCTURA DE DIETA DISOCIADA EN MICROCICLO DE COMPETICIÓN}

La distribución de los principios inmediatos que debe seguir un futbolista profesional sería la de una utilización de hidratos de carbono cercana al 55-60\%, de lípidos 25-30\% y de proteínas entre un 12-15\%, del aporte calórico total en su dieta (7), por lo que tenderemos a tener una dieta ligeramente hiperglucídica atendiendo a los vías energéticas predominantes en el fútbol.

Al comparar futbolistas que siguieron una dieta con un $65 \%$ del aporte calórico a base de hidratos de carbono con otro grupo que siguió una dieta con un $30 \%$ de hidratos de carbono, se comprobó como el primer grupo obtuvo un rendimiento mucho mejor a la hora de ejecutar carrera de alta intensidad durante el partido en comparación los jugadores cuya dieta era de un $30 \%$ de carbohidratos $(2,3)$.

En el fútbol, algunas investigaciones recomiendan aprovechar el sobradamente demostrado efecto ergogénico de la supercompensación de hidratos de carbono durante el periodo competitivo. Es decir, se busca una sobrecarga de hidratos de carbono en la dieta, durante los días previos a la competición para aumentar los depósitos de glucógeno muscular disponibles en la competición (9). No se trata de aplicar el método disociado clásico escandinavo, basado en los estudios de fisiólogos escandinavos (10-13), que consiste, en realizar 3 entrenamientos muy intensos con dieta muy pobre en hidratos de carbono, para deplecionar al máximo los depósitos de glucógeno, y así estimular la actividad de la glucógeno sintetasa. Después seguirían tres días antes de la competición en los que se entrena suave y se hace una dieta muy rica en hidratos de carbono, lo que provoca aumentos importantes en los depósitos de glucógeno muscular. Estos métodos tan agresivos, además de ser poco agradables, solían conllevar problemas como alteraciones digestivas, hipoglucemias, cetosis, irritabilidad, cansancio, entre otros.

Por este motivo se propone una estructura más moderada en la que no se produzca el vaciado completo de las reservas de hidratos de carbono. En la figura 1 se presenta la propuesta de distribución calórica basada en un método disociado moderado.

El consumo diario de hidratos de carbono que se recomienda para mantener las reservas de glucógeno muscular durante varios días de entrenamiento intenso es de 500-600 g o 8-19 g/ $/ \mathrm{kg}$ de peso, y debe suponer el total de la energía consumida. Se recomiendan alimentos con elevado contenido en almidón, para inducir una mayor síntesis de glucógeno (14-16).

\section{Distribución calórica de la dieta prepartido}

En la práctica de deportes como el fútbol donde los ejercicios son de alta intensidad desde el inicio del mismo, se recomienda un consumo previo de hidratos de carbono (17).

La literatura científica muestra que el fútbol europeo es una actividad que requiere a los depósitos de glucógeno muscular, y por tanto el volumen y la intensidad de práctica de esta modalidad deportiva está influenciada por el contenido del glucógeno muscular (18).

Siguiendo las recomendaciones todavía vigentes $(14,15)$, ha de ser una comida de fácil digestión, con alto contenido en hidratos de carbono y tres o cuatro horas antes del partido.

\section{Partido por la mañana}

Se deberá hacer un desayuno, dos o tres horas antes del partido, tomando como base el desayuno correspondiente a los días de entrenamiento, procurando incluir alimentos de estos tres grupos.

Entre 500 y 700 kilocalorías.

$\sqrt{ }$ Cereales, pan tostado, galletas, miel o mermeladas.

$\sqrt{ }$ leche o yogur, mantequilla o margarina, nosotros 
recomendamos aceite de oliva virgen extra por su alto contenido en ácidos grasos monoinsaturados. $\sqrt{ }$ fruta o zumos.

\section{Partido por la tarde}

Desayuno habitual.

Comida (Suele ser la comida más fuerte del día la mayoría de los países Latinoamericanos y en España. Se suele realizar entre las 12 y las 15 horas, según la zona geográfica): Cuatro horas antes del partido. No rebasar las 700 calorías.

Cena (última comida del día): La cena puede ser más abundante en calorías puede llegar hasta las 1500 Kcal. Es muy importante que sea especialmente rica en hidratos de carbono. El plato principal ha de ser de pasta, papas asadas o hervidas, o arroz. Se trata de reponer las reservas de glucógeno del modo más eficiente posible.

Desayuno habitual.

\section{Partido por la noche}

Comida: Preferiblemente la misma que en el apartado anterior, en cuanto a distribución de alimentos, pero puede aumentarse la cantidad de ellos, hasta llegar a unas 1000 calorías.

Merienda (comida ligera que se suele hacer por la tarde antes de la cena, en algunas zonas de Chile se denomina "once"): dos o tres horas antes del partido. A base de zumos, frutas, galletas o tostadas, miel o mermelada, yogur, café o te.

No obstante, el día anterior al partido es aconsejable insistir en una dieta más abundante en productos hidrocarbonados (arroz, pastas, patatas, frutas, mermeladas, miel, muesli, entre otros) con el fin de asegurar una mayor reserva de glucógeno. Si se acompaña ese día de un entrenamiento muy suave, para no descargar el glucógeno almacenado, se recomienda como la forma idónea de actuación.

\section{Durante el partido}

Uno de las cuestiones más investigadas en el fútbol, ha sido la hidratación y el aporte nutricional intrapartido (19-38). Teniendo presente que este tema requiere un tratamiento en profundidad, a continuación se expone una síntesis de algunas de las recomendaciones que emergen de estas investigaciones.

Normalmente la mayoría de los futbolistas no beben suficiente durante un partido, si atendemos a las pérdidas que se pueden llegar a producir. Dependiendo de las condiciones climáticas y de la intensidad del partido, las pérdidas por sudoración en futbolistas pueden ir de 1 a 4 litros (24). Por consiguiente el objetivo principal desde el punto de vista nutricional será mantener hidratado al futbolista.

Una pérdida de tan solo el $2 \%$ de el peso corporal

\section{FIGURA 1}

\section{Distribución porcentual del aporte calórico por principios inmediatos}

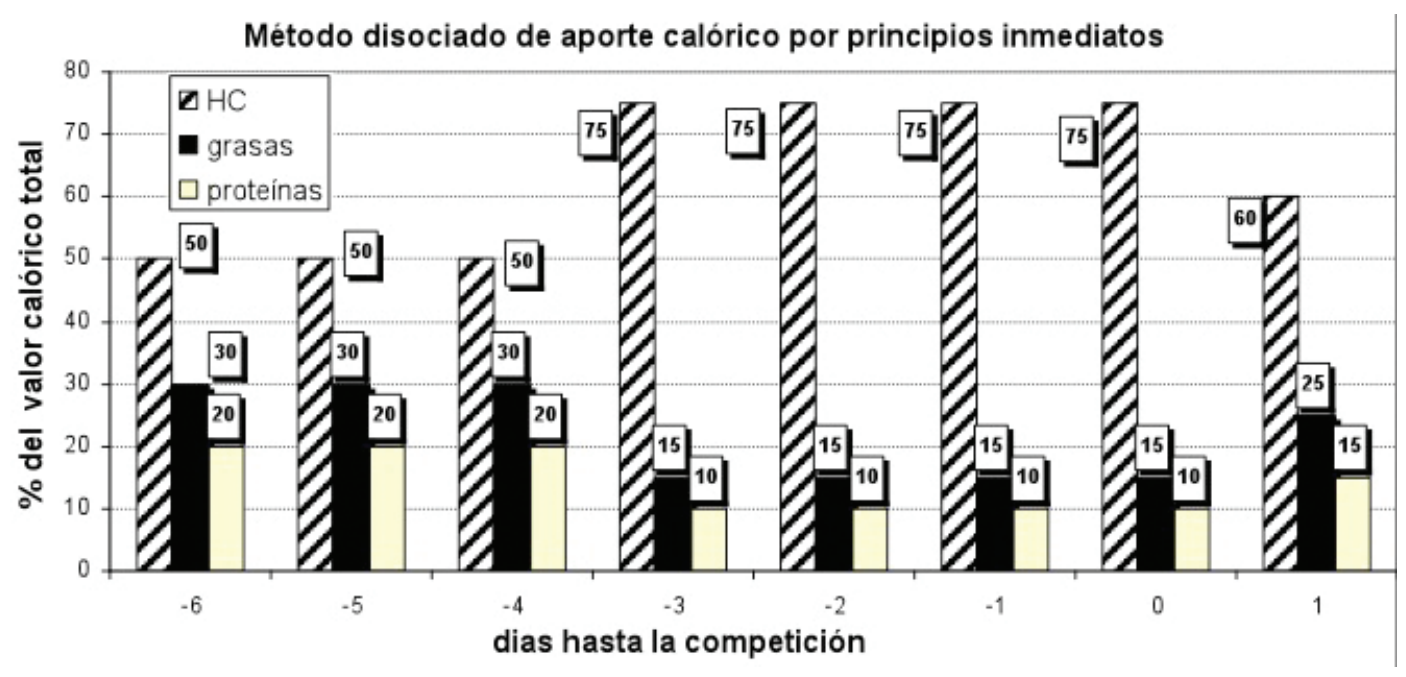


inicial (por ejemplo perder $1.4 \mathrm{~kg}$ en un deportista de 70 kilos), debido a una recuperación insuficiente de las pérdidas por sudoración puede deteriorar la carrera continua (33) o intermitente (35) y reducir el rendimiento de habilidades relacionadas con el fútbol (36).

La duda que surge es utilizar agua o bebidas deportivas. Si no disponemos de presupuesto, el agua es mejor que nada y puede ser suficiente para el entrenamiento y la competición en ambientes fríos o cuando la intensidad del partido es baja o moderada. Sin embargo, para partidos intensos y competición, las bebidas con carbohidratos y electrolitos (bebidas deportivas) son preferibles en la mayoría de los estudios realizados en fútbol $(4,29,34,38)$.

Hay varias razones por las cuales las bebidas deportivas son superiores al agua como bebida de rehidratación durante el ejercicio. Estas bebidas contienen cloruro sódico (sal de mesa) y carbohidratos como la sacarosa y glucosa. Cuando al agua se le añaden sales y carbohidratos mejora el vaciamiento gástrico, por tanto mejora su transporte del intestino a la sangre en comparación a cuando se bebe sólo agua (30).

Los carbohidratos, por supuesto, proveen energía extra, especialmente en los últimos momentos del partido. También, algunos futbolistas pierden una gran cantidad de sales por sudoración) y esta sal tiene que ser recuperada si se pretende permanecer hidratado. Además de mejorar la absorción de agua en el intestino, la sal en una bebida deportiva estimula la sed a través del cerebro y minimiza la formación de orina en los riñones, mejorando así la habilidad del cuerpo para retener el agua corporal (24).

Finalmente, cuando los deportistas calientan y están sudorosos normalmente beben más de una bebida que tenga un sabor agradable que de agua (37). La glucosa, sacarosa, fructosa, y maltodextrinas (polímero de la glucosa) son carbohidratos apropiados para componer una bebida deportiva. De hecho, debido a que cada carbohidrato mejora la absorción de líquido en el intestino por diferentes vías, tener varios tipos de carbohidratos en una bebida de rehidratación puede ser beneficioso (30). Sin embargo, la fructosa no debe de ser incluida en una bebida deportiva puesto que se absorbe lentamente en el intestino y puede producir malestar estomacal, náusea y diarrea si se ingiere en concentraciones mayores que $3-4 \%$. La concentración total de carbohidratos en una bebida deportiva debería ser de 5-7\%, esto es 5-7 gramos por cada $100 \mathrm{~mL}$ de bebida.

Concentraciones menores no producirían una mejora en el rendimiento y concentraciones mayores disminuyen el vaciado gástrico y tienden a causar problemas gastrointestinales.

\section{CONCLUSIONES}

Lo que un jugador coma y beba en el día y horas previas al partido, así como durante el propio partido, puede influir en el resultado, reduciendo los efectos de la fatiga y permitir que los jugadores desarrollen al máximo sus capacidades físicas y habilidades tácticas. Comer y beber adecuadamente poco tiempo después del partido o el entrenamiento pueden optimizar la recuperación (FIFA) (6).

Los jugadores de fútbol pueden contribuir a mantenerse sanos, evitando lesiones y logrando sus objetivos de rendimiento, adoptando buenos hábitos alimentarios. Los futbolistas han de elegir comidas que ayuden a soportar entrenamientos intensivos y optimicen el rendimiento en los partidos.

\section{RESUMEN}

Las estrategias nutricionales son uno de los pilares básicos del rendimiento en el deporte de competición. En el fútbol como en la mayoría de los deportes profesionales, el control nutricional del jugador ha cobrado una gran importancia. En este trabajo se presenta una propuesta general de la estructura de la dieta para el entrenamiento y la competición basada en la disociación calórica buscando una sobrecarga de hidratos de carbono. Estrategias nutricionales basadas en la disociación de principios inmediatos en función de las necesidades metabólicas de la competición y el entrenamiento, se han demostrado eficaces en la mejora del rendimiento de los deportistas. En el fútbol se han reportado numerosos estudios que describen las necesidades metabólicas durante un partido y durante los entrenamientos. La supercompensación de hidratos de carbono es el tipo de estrategia nutricional que ha demostrado ser más eficaz.

Palabras clave: nutrición, fútbol, dieta disociada, carbohidratos, rendimiento.

Dirigir la correspondencia a:

Sr.

José Antonio González J.

Facultad del Deporte

Universidad Pablo de Olavide de Sevilla

Carretera de Utrera Km. 1. C.P. 41013.

Sevilla.

España.

Teléfono: 0034-651517571

E-mail: jagonjur@upo.es

\section{BIBLIOGRAFÍA}

1. Bangsbo J. Energy demands in competitive soccer. J Sports Sci 1994;12:S5-s12.

2. Saltin B. Metabolic fundamentals of exercise. Med 
Sci Sports 1973;5:137-146.

3. Balsom PD, Wood K, Olsson P, Ekblom B. Carbohydrate intake and multiple sprint sports: with special reference to football (soccer). Int J Sports Med 1999;20:48-52.

4. Nicholas CW, Williams C, Lakomy HK, Phillips G, Nowitz A. Influence of ingesting a carbohydrateelectrolyte solution on endurance capacity during intermittent, high-intensity shuttle running. J Sports Sci 1995;13:283-290.

5. Krustrup P, Mohr M, Steensberg A, Bencke J, Kjaer M, Bangsbo J. Muscle and Blood Metabolites during a Soccer Game: Implications for Sprint Performance. Med Sci Sports Exerc 2006;38:1165-1174.

6. FIFA. Nutrition for football: The FIFA/F-MARC Consensus Conference. J Sports Sci 2006;24:663664.

7. Leblanc JC, Le Gall F, Grandjean V, Verger P. Nutritional intake of French soccer players at the Clairefontaine Training Center. Int J Sport Nutr Exerc Metab 2002;12:268-280.

8. Ruiz Ft, Irazusta A, Gil S, Irazusta J, Casis L, Gil J. Nutritional intake in soccer players of different ages. J Sports Sci 2005;23:235-242.

9. González-gallego J, Villa J: Nutrición y ayudas ergogénicas en el deporte. ed Síntesis, Madrid, 2001.

10. Bergstrolm J, Hermansen L, Hultman E, Saltin B. Diet, muscle glycogen and physical performance. Acta Physiol Scand 1967;71:140-150.

11. Gollnick PD, Armstrong RB, Sembrowich WL, Shepherd RE, Saltin B. Glycogen depletion pattern in human skeletal muscle fibers after heavy exercise. J Appl Physiol 1973;34:615-618.

12. Gollnick PD, Piehl K, Saubert 4th CW, Armstrong RB, Saltin B. Diet, exercise, and glycogen changes in human muscle fibers. J Appl Physiol 1972;33:421425.

13. Hermansen L, Hultman E, Saltin B. Muscle glycogen during prolonged severe exercise. Acta Physiol Scand 1967;71:129-139.

14. Maughan RJ. Energy and macronutrient intakes of professional football (soccer) players. Br J Sports Med 1997;31:45-47.

15. Maughan RJ, Shirreffs SM. Nutrition for Soccer Players. Curr Sports Med Rep 2007;6:279-280.

16. Costill DL. Carbohydrates for exercise: dietary demands for optimal performance. Int J Sports Med 1988;9:1-18.

17. MacMillan N. Ejercicio y quema de grasa: ¿comer o no comer antes de entrenar? Rev Chil Nutr 2009;36:72-74.

18. Shephard RJ. Meeting carbohydrate and fluid needs in soccer. Can J Sport Sci 1990;15:165-171.

19. Ali A, Williams C, Nicholas CW, Foskett A. The influence of carbohydrate-electrolyte ingestion on soccer skill performance. Med Sci Sports Exerc 2007;39:1969-1976.

20. Clarke ND, Drust B, MacLaren DPM, Reilly T. Strategies for hydration and energy provision during soccer-specific exercise. Int J Sport Nutr Exerc Metab 2005;15:625-640.

21. Ebine N, Rafamantanantsoa HH, Nayuki Y, Yamanaka K, Tashima K, Ono T, Saitoh S, Jones PJH. Measurement of total energy expenditure by the doubly labelled water method in professional soccer players. J Sports Sci 2002;20:391-397.

22. Kirkendall DT. Fluid and electrolyte replacement in soccer. Clin Sports Med 1998;17:729-738.

23. Leiper JB, Prentice AS, Wrightson C, Maughan RJ. Gastric emptying of a carbohydrate-electrolyte drink during a soccer match. Med Sci Sports Exerc 2001;33:1932-1938.

24. Maughan RJ, Merson SJ, Broad NP, Shirreffs SM. Fluid and electrolyte intake and loss in elite soccer players during training. Int. J Sport Nutr Exerc Metab 2004;14:333-346.

25. Maughan RJ, Shirreffs SM. Nutrition and hydration concerns of the female football player. Br J Sports Med 2007;41.

26. Maughan RJ, Shirreffs SM, Merson SJ, Horswill CA. Fluid and electrolyte balance in elite male football (soccer) players training in a cool environment. J Sports Sci 2005;23:73-79.

27. Maughan RJ, Watson P, Evans GH, Broad N, Shirreffs SM. Water balance and salt losses in competitive football. Int J Sport Nutr Exerc Metab 2007; 17:583-594.

28. Newell M, Newell J, Grant S. Fluid and electrolyte balance in elite gaelic football players. Ir Med J 2008; 101 .

29. Ostojic SM, Mazic S. Effects of a carbohydrateelectrolyte drink on specific soccer tests and performance. J Sports Sci Med 2002;1:47-53.

30. Shi X, Summers RW, Schedl HP, Flanagan SW, Chang R, Gisolfi CV. Effects of carbohydrate type and concentration and solution osmolality on water absorption. Med Sci Sports Exerc 1995;27:16071615.

31. Shirreffs SM, Sawka MN, Stone M. Water and electrolyte needs for football training and match-play. J Sports Sci 2006;24:699-707.

32. Siegler JC, Mermier CM, Amorim FT, Lovell RJ, McNaughton LR, Robergs RA. Hydration, thermoregulation, and performance effects of two sport 
drinks during soccer training sessions. J Strength Conditioning Res 2008;22:1394-1401.

33. Armstrong LE, Costill DL, Fink WJ. Influence of diuretic-induced dehydration on competitive running performance. Med Sci Sports Exerc 1985;17:456461.

34. Leatt PB, Jacobs I. Effect of glucose polymer ingestion on glycogen depletion during a soccer match. Can J Sport Sci 1989;14:112-116.

35. Maxwell NS, Gardner F, Nimmo MA. Intermittent running: muscle metabolism in the heat and effect of hypohydration. Med Sci Sports Exerc 1999;31:675683.
36. McGregor SJ, Nicholas CW, Lakomy HKA, Williams $\mathrm{C}$. The influence of intermittent high-intensity shuttle running and fluid ingestion on the performance of a soccer skill. J Sports Sci 1999;17:895903.

37. Passe DH, Horn M, Stofan J, Murray R. Palatability and Voluntary Intake of Sports Beverages, Diluted Orange Juice, and Water During Exercise. Int J Sport Nutr Exerc Metab 2004;14:272-284.

38. Welsh RS, Davis JM, Burke JR, Williams HG. Carbohydrates and physical/mental performance during intermittent exercise to fatigue. Med Sci Sports Exerc 2002;34:723-731. 